\title{
La quantification dissociée dans les indéfinis Free Choice du français
}

Claude Muller (Université de Bordeaux et CNRS, UMR 5263 - CLLE)

Langue Française 166, juin 2010, 93-107.

abstract

In this paper, we examine how the different free-choice determiners and pronouns in French differ in their quantificational properties, between universal or existential interpretations, what could be described in analysing actions as semantic compounds, where the universal value only applies on one stage of the event: choice, presupposition, preceding or accompanying the action; or, with a reversed order, with qualifying, mostly depreciative values

\section{résumé}

Dans cet article, on examine comment les termes de libre choix du français varient selon les contextes et selon les séries, dans leurs propriétés de quantification, entre une interprétation universelle et des valeurs existentielles, qui peuvent être décrites par le prise en compte de la complexité des actions modalisées. La valeur universelle ne s'applique qu'à une phase de l'événement, choix, présupposé, avec une phase de choix précédant l'action, et une phase existentielle; ou bien en ordre inverse, avec des interprétations qualificatives la plupart du temps dépréciatives.

mots-clés : free choice, quantification, polarité, indéfini. / free choice, quantification, polarity, indefinite.

\section{Les indéfinis « de libre choix » parmi les indéfinis}

On peut admettre que être indéfini, c'est une propriété sémantique des groupes nominaux, telle que ces termes n'ont pas, indépendamment de la prédication dont ils sont des actants, une référence stable, préétablie et réutilisable. Leur référence est obtenue par le croisement du sens propre du groupe nominal avec le sens induit par le rôle d'argument de la prédication qui les introduit. Des termes comme : cinq personnes, beaucoup d'étudiants, des ouvriers, la plupart des participants, n'ont pas de référence autonome. Si on se limite aux indéfinis comptables, on peut voir en un groupe nominal indéfini une énumération disjonctive d'unités de même contenu sémantique. Si la prédication est une proposition à valeur générale ou générique, on en reste à ce statut :

(1) Cinq personnes rassemblées dans un lieu public forment déjà une manifestation au regard de la loi.

En (1), la prédication verbale s'applique à tout groupe nominal « cinq personnes rassemblées dans un lieu public », mais elle ne spécifie aucun d'entre eux. Si par contre la prédication singularise une de ces unités, elle isole cette unité parmi les autres et permet une identification définie ultérieure :

(2) Cinq ouvriers ont manifesté en brandissant des pancartes. Les cinq ouvriers ont été emmenés au commissariat.

La spécification ne fait donc pas partie de la définition des groupes nominaux indéfinis. Cette propriété dissocie certains types d'indéfinis parmi d'autres. La spécification est possible avec la plupart des indéfinis couramment utilisés dans les assertions affirmatives : c'est pour eux une affaire de contexte. Par contre, il n'y a pas de spécification possible avec certaines catégories d'indéfinis comme les négatifs (personne, aucun) parce que le contexte de leur association à la négation leur enlève cette possibilité. On va voir que la spécification est également exclue pour les indéfinis « de libre choix ». 


\section{Les indéfinis 'free choice'}

Les indéfinis " de libre choix» ou 'free choice' (en abrégé FC) sont caractérisés par un blocage des possibilités de spécification: des termes comme la série qui/ quoi que ce soit, n'importe qui/ quoi, ou encore un ...quelconque, comportent en plus de l'indéfinition basique (pour les deux premières séries, celle attachée au relatif/interrogatif ${ }^{1}$ quel et ses variantes pronominales qui /quoi), une expansion prédicative qui est soit un autre verbe figé n'importe, soit une relative à effet élargissant que ce soit, soit un adjectif signifiant l'absence de singularité, quelconque. Ce qui les distingue des indéfinis qui sont soit spécifié, soit non spécifiés, comme les articles un, des, les pronoms quelqu'un, quelque chose, ou les numéraux, c'est que le contenu sémantique de cette prédication incluse dans l'indéfini bloque d'une façon ou d'une autre la spécification entendue comme le repérage d'un groupe nominal distingué des autres de même sens du seul fait qu'il est argument de la prédication verbale.

Il s'agit d'une catégorie d'emplois déterminée empiriquement, d'abord chez Vendler 1967, qui parle de termes pour lesquels il y a freedom of choice, "liberté de choix »; sous l'étiquette free choice, ils apparaissent chez Ladusaw 1980, puis Carlson 1981. La catégorie, très empirique, désigne essentiellement les items du type de any en dehors des contextes à polarité négative ${ }^{2}$. La série de type ${ }^{3}$ n'importe représente le mieux cette catégorie en français, dans des contextes où d'autres indéfinis ne seraient pas utilisables, comme par exemple dans :

\section{Il ferait n'importe quoi pour réussir.}

La frontière avec la polarité n'est pas toujours très nette, et il n'est pas exclu qu'une mise à plat de l'ensemble de cette question conduise à des reclassements. On a une idée de cette démarche si on lit le travail d'Haspelmath 1997, conduit dans une perspective typologique et de comparaison des langues : la notion de polarité disparaît, comme étant un concept trop général, pour faire place à des sous-catégories fonctionnelles d'emplois, qui sont déterminées empiriquement. Cependant, la catégorie des free choice est distinguée de l'ensemble des souscatégories relevant de la polarité négative ; comme celles-ci, et comme les emplois de type spécifié mais dans des domaines modaux (hors du réel), elle forme une des trois catégories d'emplois « non spécifiques » de sa terminologie.

On peut définir comme suit ces indéfinis:

Les indéfinis de libre choix caractérisent des groupes nominaux comportant des objets qui sont et restent interchangeables et indifférenciés dans leur ensemble de référence, sans que leur utilisation comme arguments d'un prédicat leur donne une référence spécifiée.

La propriété essentielle est donc celle de la non spécification, due au contenu même du groupe nominal : il y a interchangeabilité entre les items de même contenu. Le caractère interchangeable des termes peut lui-même être motivé sémantiquement, ou peut-être pragmatiquement, par des considérations diverses, par exemple selon Vlachou : l'ignorance, l'indifférence, l'absence de choix, l'indistinguabilité, le jugement dépréciatif ('low-level' chez Vlachou). Dans certains cas, il faut cependant préciser que l'indéfini 'free choice' est un masque qui cache pour diverses raisons un objet spécifique, éventuellement réel, comme on le verra plus loin.

\footnotetext{
${ }^{1}$ Les formes quelque, quelconque sont historiquement issues de quel. On trouve par conséquent une base morphologique identique pour les trois séries. Sur le caractère indéfini de toutes les formes Qu-, voir Muller 2008.

${ }^{2}$ Voir Vlachou 2007, chapitre 2, pour un historique montrant l'intrication de cette notion avec la polarité négative. Voir aussi Muller 2006, 2007.

${ }^{3}$ L'étude de Paillard, 1997, est une des toutes premières à s'intéresser exclusivement à ces termes. Dans les perspectives actuelles d'analyse, voir Jayez \& Tovena 2005.
} 
La non-spécification en soi ne suffit pas à assurer la propriété d'interchangeabilité ou d'indifférenciation. R. Martin 1992 signalait qu'avec un exemple comme (4), il y a non spécificité (possible) si le sujet de la principale forme un souhait abstrait ${ }^{4}$, sans qu'il y ait libre choix :

Pierre veut épouser une Portugaise (Martin 1992 : 176)

Cela ne signifie pas qu'il souhaite épouser n'importe quelle Portugaise. Cela n'est pas impossible, mais le sens est différent et le groupe nominal doit recevoir dans ce cas une prédication portant le sens de libre choix :

(5) Pierre veut épouser n'importe quelle Portugaise, pour qu'elle lui donne la nationalité portugaise dont il a besoin.

L'exemple (5) fait apparaître un des aspects surprenants de ces indéfinis. D'abord, le caractère fugace de la qualification: une fois épousée, la Portugaise ne sera plus «n'importe quelle Portugaise ». En contexte factif, la caractérisation FC est peu acceptable :

(5') ??Pierre a épousé n'importe quelle Portugaise, afin qu'elle lui donne la nationalité portugaise dont il a besoin.

En effet, le contexte factif fait de l'indéfini un élément spécifié, même s'il est inconnu du locuteur. Par contre " une quelconque Portugaise» passera mieux, avec une interprétation légèrement différente : soit celle de l'indifférence quant à la spécification précise, soit encore un jugement plutôt dépréciatif.

Une propriété qu'on va examiner plus précisément dans cet article est celle de l'interprétation de la quantification. La quantification réelle, liée à la pragmatique de l'énoncé, est souvent masquée par la prédication exprimant le libre-choix. En (5), la valeur ne peut être que le singulier, le verbe ne se prêtant pas à une utilisation itérative dans nos sociétés. On remarquera que cela exclut l'autre série courante dans des emplois $\mathrm{FC}$, la série que ce soit :

*Pierre veut épouser quelque Portugaise que ce soit.

parce que dans ce cas, la quantification tend à prendre une valeur universelle, non seulement pour le choix, mais pour l'action qui suit le choix : si X est Portugaise, alors Pierre veut l'épouser (ce qui vaut aussi pour n'importe quelle Portugaise) mais de plus, l'action n'est pas bornée et prend une interprétation itérative : «chaque fois qu'il rencontre une Portugaise, il veut l'épouser $»$. On voit donc surgir une question intéressante, qui permet de différencier les emplois des FC : avec certains d'entre eux, le libre choix porte sur une phase qui précède l'action, et la quantification (au sens pragmatique : combien de fois l'action a lieu, combien d'items indéfinis sont des arguments de l'action) est indépendante de cette phase du choix. Avec d'autres, au contraire, les phases de choix et d'action mettant en jeu les variables de l'ensemble représenté par l'indéfini sont liées.

Il faut noter cependant que la série n'importe n'interdit pas l'itération :

N'importe quel étudiant peut se présenter à cet examen

\footnotetext{
${ }^{4}$ Il y a quand même spécification, si on veut, mais elle reste cantonnée dans le domaine modal de la prédication : La Portugaise qu'il veut épouser doit parler le français et mesurer au moins 1 m80...Dans la littérature, on parle uniformément d'emplois non spécifiques des indéfinis. Je préfère, dans ce cas, parler de spécifiés non spécifiques, puisque la spécification existe bien dans un monde non réel.
} 
(7) Prends n'importe quel tournevis dont tu auras besoin!

En (6), la quantification peut être décrite comme universelle (sur un ensemble borné). En témoigne la possibilité d'utiliser tout avec le même sens, ce terme n'ayant que cette possibilité d'utilisation ici:

(6’) Tout étudiant peut se présenter à cet examen.

La quantification est encore différente en (7) : l'impératif offre le libre choix d'un, mais peutêtre aussi de plusieurs, parmi les tournevis présents dans le contexte. La quantité attachée à l'action va de un à la totalité de ce qui est disponible.

Nous allons examiner comment fonctionne la quantification dans les deux séries les plus représentatives des FC en français, n'importe et que ce soit.

3. Le rapport à la quantité sans dissociation entre sélection et action

Dans un certain nombre de cas, l'indéfini de libre choix ne dissocie pas le moment du choix de celui de l'action. L'extension est la même et vaut pour la totalité de l'ensemble de référence. Les formes de type n'importe ou que ce soit sont remplaçables par tout ou l'équivalent (par exemple quiconque pour les personnes). De plus, on peut utiliser avec le même sens dénotationnel des indéfinis qui sont plus nettement des sommes additives plutôt que des disjonctions (tous les) :

(8) Toute chouette chasse des souris (idem avec n'importe quelle. Même dénotation avec toutes les).

(9) Quelque fraudeur que ce soit sera puni

On y trouve les emplois en phrase générique, les emplois modaux à valeur générale (qui sont des équivalents de systèmes hypothétiques à application automatique : si $x$ (ou y) est un fraudeur, il sera puni. On peut y voir le domaine propre des FC. Les comparatives d'inégalité y sont assimilables :

(10) Il réussit mieux que personne / tout le monde / qui que ce soit /n'importe qui / quiconque.

On peut y ajouter des emplois non génériques dans lesquels l'indéfini a également une extension maximale (non bornée) comme ${ }^{5}$ :

(11) Pour qui que ce soit, nous n'avons pas mis les pieds ici aujourd'hui, comprends-tu?

(G. Bernanos, Nouvelle histoire de Mouchette, 1937)

(12) Je défie qui que ce soit de m'apporter la preuve du contraire.

Ici aussi, le remplacement par tout semble possible sans changement de sens. Enfin, l'emploi additif semble assez proche (défier tout le monde, en contexte borné, signifie défier tous les gens ici présents). On trouve des emplois de ce type avec tout comme avec n'importe quel:

(13) Je signale à mon médecin tout malaise survenant à l'effort. (Journal municipal, Bègles, janvier 2010)

\footnotetext{
${ }^{5}$ Cet exemple comme tous ceux qui suivent tirés de textes littéraires datés provient de la base Frantext.
} 
Dans les phrases non habituelles factives ${ }^{6}$, on peut utiliser $n$ 'importe sur un ensemble borné pragmatiquement, et l'effet obtenu est alors additif :

(14) N'importe quel élève de cette classe a bien rendu un devoir !

(= tous les élèves)

\section{Les emplois à dissociation}

Dans de nombreux cas, avec l'une ou l'autre des séries de libre choix, on doit comprendre que la quantification sous-jacente n'est pas équivalente à la quantification universelle. Par exemple, dans l'emploi ci-dessous, pourtant assez proche apparemment des précédents :

(15) Avoir pour qui ou quoi que ce soit une admiration sans bornes -c'est être borné par cette admiration (P. Reverdy, Le livre de mon bord 1936)

Il n'y a pas d'équivalent additif : on ne peut pas, en gardant le même sens dénotatif, remplacer l'indéfini par une expression comme tout le monde ou tous les hommes, même si toute personne semble possible ici : autrement dit, la suite disjonctive une personne ou une autre personne ne prend pas un sens additif. La quantité visée est singulière: une personne ou une chose, quelle qu'elle soit. Le choix de l'indéfini n'est donc pas lié à la quantification de l'action "avoir pour x une admiration sans bornes » mais à l'indifférenciation qualitative de l'argument de cette action, donc le «choix» de l'argument. On remarquera qu'ici l'indifférenciation repose sur deux critères distincts: d'une part, une possible échelle de valeurs (presque toujours discernable avec que ce soit) : "même pour la personne la plus digne d'admiration », par exemple. D'autre part, par le caractère sinon générique, du moins général de l'énoncé, présenté comme une maxime applicable dans toute situation semblable. Les exemples ci-dessous, qui sont des phrases résumant un ensemble de situations répétées, présentent la même double indifférenciation, également avec une possible interprétation scalaire : chaque fois, une seule valeur de la variable représentée par l'indéfini est instanciée, mais d'une part, le repérage de cette valeur est signalé comme n'ayant aucune importance, et d'autre part, la répétition de l'action justifie l'absence de pertinence de ce repérage.

(16) ...quand elle dit ce qu'elle pense de quoi que ce soit...est-elle capable de penser? (N. Sarraute, Enfance, 1983)

(17) Qu'on fasse allusion à quoi que ce soit au sujet de son père le met de mauvaise humeur. (M. Charef, Le thé au harem d'Archi Ahmed, 1983)

D'autres contextes offrent des situations différentes : par exemple, en situation d'énumération dans un contexte événementiel :

(18) Ils regardaient la bière ou leur canne, ou n'importe quoi, mais ils ne regardaient que cela. (A. Camus, L'étranger, 1942).

On peut admettre que l'indéfini signale un objet singulier, mais différent pour chaque individu du groupe. La position de l'indéfini est à la fin de l'énumération disjonctive, et renvoie à une série de concrétisations possibles de cette variable singulière, avec la même idée : la valeur de cette variable n'a aucune importance. On notera une différence quand même: la

\footnotetext{
${ }^{6}$ Les formes tout et que ce soit ne sont généralement pas utilisables telles quelles dans ces contextes.
} 
quantification n'offre ici aucun trait de scalarité, autrement dit, les choses regardées ne sont pas organisées sur une échelle de valeurs quelconque les ordonnant l'une par rapport à l'autre. Egalement dans un contexte événementiel, l'exemple suivant illustre la répétition d'une action, mais sans simultanéité.

(19) En effet, toutes les cinq chansons, l'animateur appelle n'importe qui, au hasard du Bottin, afin qu'il lui cite le montant exact de la somme en jeu. (Ernaux, Journal $d u$ dehors, 1993)

Il est évident que chaque fois l'indéfini signale une seule personne, et que la valeur de l'argument n'entre pas en compte, étant expressément signalée comme sans importance («au hasard du Bottin »). L’indéfini est spécifié non spécifique, comme en (5), avec le brouillage supplémentaire de la répétition. Le libre choix correspond à l'univers antérieur à l'action, celui de la sélection d'un individu, alors que l'action affecte strictement une seule personne. On notera aussi qu'il n'y a pas de scalarité envisageable ici, ce qui semble bien être un critère déterminant dans le choix de l'indéfini utilisé, n'importe plutôt que que ce soit.

On retrouve ces phénomènes de dissociation dans un des contextes typiques des emplois de libre choix : celui des impératifs. L’impératif suppose une spécification hors du réel, puisque l'action n'est pas encore accomplie. L'interprétation de la quantité de l'argument objet tient à des facteurs pragmatiques, la possibilité ou non de répéter l'action, ou d'affecter un nombre pluriel d'objets. Le libre choix, donc la suite disjonctive ouverte, se limite à la phase de choix précédant l'action :

(20) Fais n'importe quoi à déjeuner (Colette, Chambre d'hôtel, 1940)

Prenez n'importe quelle carte !

La quantité concernée par l'action est évidemment existentielle ici, mais il est usuel que dans un tour de carte on se limite à une seule carte, alors que dans la préparation d'un repas, le nombre de plats peut être pluriel. Il n'est d'ailleurs pas exclu de trouver ici la quantification universelle :

(22) Confisquez n'importe quel alcool! (exemple traduit de Dayal, 2004 )

ou encore un choix ouvert pouvant s'appliquer à un nombre indifférencié d'objets (éventuellement tous) :

Prends n'importe quel outil dont tu as besoin !

On le voit sur tous ces exemple, la quantification dissociée peut s'analyser comme un choix ouvert à toute valeur de la série d'objets représentés par l'indéfini, ce choix étant indépendant de la quantification réelle (celle de l'action) masquée par cet indéfini. Dans les contextes modaux, comme dans l'impératif, le libre choix s'applique à la phase préliminaire à l'action. Dans les énumérations, c'est un peu différent : on a affaire à une série d'actions simultanées, distribuées chacune par les protagonistes. L'indéfini ne signale pas un choix antérieur à l'action, mais une somme de valeurs indifférenciées et non ordonnées: l'absence de pertinence du choix est conjugué avec le brouillage dû à la répétition au-delà de ce qui est dit du même processus. Sans répétition, pour une action épisodique factive, l'effet n'est pas le même :

?A ce moment-là, l'homme regardait n'importe quoi. 
La phrase est moins acceptable, et si on l'accepte, c'est avec un sens différent : celui de la qualification, qui se fait a posteriori, comme sans pertinence ni intérêt de l'identité de l'objet regardé. Il y a plus nettement un emploi qualificatif et attributif, souvent chargé de connotations plutôt dépréciatives. On examinera ces emplois plus loin.

Dans les actions répétées non simultanées, on observe le même effet de brouillage de l'identification des objets qui est produit par la répétition. Il peut y avoir une prédication explicite de l'absence de pertinence d'un choix quelconque d'argument, comme en (19) mais souvent, le brouillage tient à la seule répétition. Dans ces contextes, la présentation groupée, en un seul objet linguistique, d'un ensemble non ordonné de valeurs diverses de la variable, liées chacune à un des événements répétitifs mentionnés, abolit toute possibilité de spécification, donc à la prédication n'importe.

\section{La quantification dissociée dans les contextes à polarité}

Dans la dépendance de la négation, l'utilisation des formes neutres des indéfinis, comme quelqu'un, quelque chose, risque d'être mal interprétée: on peut y voir l'utilisation d'un indéfini spécifié hors de la portée de la négation :

(25) Je n'ai pas parlé à quelqu'un (= il y a quelqu'un à qui je n'ai pas parlé, et non : je n'ai parlé à personne) $)^{7}$.

On utilise souvent des séries spécialisées d'indéfinis (ici, pour le français, personne) mais également les séries exprimant le libre choix, lorsqu'elles ne risquent pas d'interférer avec la négation : ainsi n'importe est acceptable seulement dans les contextes de négation indirecte, mais pas dans le contact immédiat avec la négation : dans ce dernier cas, le prédicat interne est la cible de la négation, et c'est précisément la liberté de choix qui est niée. On aura par exemple la possibilité de dire avec à peu près le même sens qu'un mot négatif:

(26) Je ne pense pas que n'importe qui vienne nous voir

(27) Si elles échouent aussi déplorablement que l'organisation qui les a précédées, aucun succès dans n'importe quel autre domaine ne pourra compenser cette faiblesse fondamentale. (Charte des Nations Unies : statut de la Cour internationale de justice, 1946)

mais dans :

On ne recevra pas n'importe qui

le sens est différent : on recevra certaines personnes sélectionnées.

L'utilisation de la série de libre choix que ce soit exclut toute interprétation de ce type ${ }^{8}$, et peut s'utiliser aussi bien dans les contextes de négation directe que de négation indirecte :

\footnotetext{
${ }^{7}$ Dès que l'indéfini n'est plus dans la portée immédiate de la négation, il redevient cependant utilisable avec le même sens que la forme que ce soit: Je ne crois pas qu'il ait eu le temps d'en parler à quelqu'un (= qui que ce soit).

${ }^{8}$ L'absence d'effet sémantique de la négation sur la prédication interne (la partie que ce soit) tient peut-être à la morphologie : la prédication est une relative, alors qu'avec n'importe, il s'agit d'un verbe ayant aussi le pronom $\mathrm{Qu}$ - comme argument. Il se peut qu'il y ait simplement une exploitation par la langue de l'existence de deux séries : l'anglais any, malgré son opacité morphologique (mais souvent renforcé par just), peut être la cible d'une négation : a trout is not (just) any fish « une truite n'est pas n'importe quel poisson » (Horn $2000: 159$ ).
} 
Quelles peuvent être les raisons qui permettent à des indéfinis de libre choix d'être utilisés dans les phrases négatives? Pour le comprendre, il faut comprendre ici aussi comment fonctionne la quantification sous les aspects pragmatiques soulignés plus haut: la phrase négative est un moyen de signifier la quantité nulle, ce qui passe par deux solutions qui sont équivalentes dans les contextes à polarité englobant la négation. Dans ces contextes, l'orientation scalaire est monotone-décroissante (Ladusaw 1980) : la négation d'une quantité renvoie à une quantité moindre, donc la négation d'un objet singulier quelconque signifie la quantité nulle (pas un signifie donc normalement «zéro » et non «plusieurs »). Par ailleurs, on peut aussi imaginer que pour tout item indifférencié envisageable ${ }^{9}$, la prédication est négative :

Personne n'est venu $=$ pour toute personne envisageable, on peut dire qu'elle n'est pas venue.

Les deux formulations (la négation d'un item isolé vu comme l'extrémité basse d'une échelle de quantité, et d'autre part la quantification universelle sur une prédication négative) sont logiquement équivalentes. Il se trouve que la série que ce soit peut entrer dans les deux points de vue, parce que son sens convient aussi bien à l'interprétation qu'on peut qualifier d'existentielle (le renvoi à un individu quelconque sur lequel porte la négation) qu'à celle, universelle, qui fait défiler tout individu de l'ensemble dans une prédication verbale négative. Sur un plan plus pragmatique, il n'est pas tout à fait exact de poser qu'il y a toujours équivalence : ainsi, la version existentielle de la quantification dans les négations est souvent attachée à l'absence d'existence dans le réel :

\section{Il n'entendait aucun bruit}

Cette phrase se comprend normalement comme l'assertion d'une absence de bruits dans le contexte (il n'y a aucun bruit à entendre), alors qu'elle peut tout à fait signifier qu'étant sourd, le sujet de l'action n'entendait aucun des bruits présents dans le contexte, même les plus forts : « tous les bruits, il ne les entendait pas ». L'interprétation universelle des phrases négatives est plus consistante si elle quantifie sur de l'existant ${ }^{10}$.

Cependant, la possible utilisation de la série n'importe, à la place d'un mot négatif ou de que ce soit, comme en (26), (27), en contexte de dépendance indirecte d'une négation, montre que ce qui est commun aux deux séries de 'free choice', l'élargissement à tout le domaine envisageable, suffit dans une certaine mesure à permettre un emploi à valeur identique tel que n'importe ne soit pas interprété avec son sens propre, mais simplement comme une suite disjonctive à interprétation universelle ${ }^{11}$.

\footnotetext{
${ }^{9}$ Avec une extension indéfinie aux valeurs extrêmes, on obtient ce que Kadmon \& Landman 1993 appellent 'strengthening', un 'renforcement' dans le virtuel de l'extension de l'ensemble de référence.

${ }^{10}$ Avec cet exemple : il n'entendait pas quelque bruit que ce soit, l'interprétation universelle est plus accessible que celle de l'absence de bruits, semble-t-il. En général, il est quand même difficile d'utiliser les FC que ce soit avant la négation : ?Qui que ce soit n'est pas venu ! même s'il en existe des attestations par exemple dans l'exemple réel suivant (en contexte modal il est vrai) : Quelque catégorie que ce soit ne peut pas être autorisée à prendre les français pour otages... (Télévision : A2, 17/02/84).

${ }^{11}$ Les exemples qui suivent montrent que $n$ 'importe s'adapte aussi bien aux contextes à organisation scalaire, comme ceux à polarité négative, qu'aux constructions typiques du libre choix, sans aucune organisation de ce type.
} 
Dans d'autres contextes à polarité que la négation, on peut retrouver des phénomènes de dissociation de la quantification, liés à une interprétation existentielle pragmatique de la quantité.

Par exemple, dans les hypothétiques :

(32) Si quoi que ce soit / n'importe quoi vous irrite, signalez-le nous pour que nous puissions y remédier.

L'interprétation est existentielle (s'il arrive qu'une chose, quelle qu'elle soit, vous irrite), et l'élargissement universel tient à la prise en compte, dans ce domaine virtuel, de tout ce qui pourrait entrer comme argument dans ce prédicat hypothétique. L'interprétation universelle est ici très différente, par exemple dans :

(33) Si quoi ce que ce soit (= tout) vous irrite, vous feriez mieux de rester chez vous.

On peut y voir, à la manière de Fauconnier (1975) ou encore Haspelmath (1997: 116) les deux pôles d'un ensemble organisé de façon scalaire. La série que ce soit, qui dénote un élément quelconque d'un ensemble, peut s'interpréter comme l'extrémité basse d'une échelle des quantités dans le contexte à polarité (donc renvoyer, selon le contexte, à une interprétation existentielle évoquant une ou peut-être quelques occurrences possibles) ou, inversement, à l'extrémité haute d'une échelle et prendre un sens universel (ce qui signifie une addition des valeurs de la variable pour l'action hypothétique). Dans l'interprétation existentielle à polarité, il y a bien ce phénomène de dissociation entre une interprétation existentielle, bornée, de la quantification, et une interprétation universelle qui est ici le libre choix offert à l'interlocuteur de la ou des choses qui pourraient l'irriter.

Il n'est d'ailleurs pas exclu de trouver ce phénomène dans un contexte à polarité faible, où l'interprétation existentielle de l'indéfini est ancrée dans le monde réel :

(34) Ces deux facteurs rendent l'ensemble hautement instable et je suis même étonné que quoi que ce soit fonctionne. (dans Vlachou 2007 : 194)

L'interprétation est existentielle ${ }^{12}$ : je suis étonné qu'il y ait quelque chose qui fonctionne. L'élargissement à l'ensemble de référence est possible parce que le verbe comporte une présupposition négative forte sur son complément : je m'attendais à ce que rien ne fonctionne. En somme, la quantification universelle porte sur l'univers du présupposé (quoi que ce soit, ça ne doit pas fonctionner). La dissociation est encore antérieure en ce sens à la réalisation de l'action verbale. Il en va de même dans des questions à forte orientation vers une réponse négative :

(35) Pourquoi serions-nous émus de quoi que ce soit? (Claudel, Tête d'or)

(36) Tu n'as rien à savoir, idiote, qui t'a demandé de savoir quoi que ce soit? (B.-M. Koltès, Quai Ouest, 1985)

Ici aussi, la quantification est existentielle; il ne s'agit pas d'être ému de tout, ni de tout savoir, mais de l'éventualité d'être ému par une chose ou une autre, ou de savoir quelque chose. La signification de quantifieur universel ne se justifie que dans l'univers du présupposé négatif («nous n'avons pas à être émus de quoi que ce soit», «on ne te demande pas de savoir quoi que ce soit »).

\footnotetext{
${ }^{12}$ D'après ce qu'en dit Vlachou (2007 : 195), l'interprétation existentielle ne fait aucun doute. Si on voulait donner une interprétation universelle assurée à la quantification, il faudrait utiliser tout ici.
} 


\section{Les deux séries et la dissociation}

Si on examine en détail les emplois des deux séries majeures de FC du français, que ce soit et n'importe, on voit qu'elles ne sont absolument pas interchangeables, même si elles partagent des domaines communs: les emplois universels, les emplois dans les constructions comparatives, et dans une certaine mesure les emplois dans les contextes à polarité de la négation indirecte et des constructions conditionnelles (cf. Muller 2009, pour une révision de la classification proposée par Haspelmath 1997). On ne trouve pas de façon naturelle et équivalente n'importe en contexte de négation directe, comme on l'a vu ci-dessus. On ne peut pas non plus utiliser cette série dans les questions à réponse négative attendue :

??Pourquoi serions-nous émus de n'importe quoi?

??Qui t'a demandé de savoir n'importe quoi ?

En revanche, lorsqu'on sort nettement des contextes à polarité, il est généralement exclu d'utiliser la série que ce soit. Par exemple, dans les contextes modalisés de type irréel non spécifique, comme les impératifs :

*Fais quoi que ce soit à déjeuner !

On a montré ailleurs ce qui fait la spécificité de chacune des deux séries (Muller 2006, 2007). La série que ce soit n'est utilisable hors des contextes universels que lorsque la quantification sous-jacente, non universelle donc, est organisée de façon scalaire, si bien que dans un contexte à polarité, que ce soit puisse représenter l'extrémité basse d'une échelle de quantité. les exemples d'emploi marginaux le montrent bien, comme celui-ci :

(40) (votre argent) Il vaut mieux le mettre dans une banque sérieuse, lui faire produire quoi que ce soit. (T. Bernard, Monsieur Codomat, 1907).

Le sens ne peut être ici qu'existentiel. L'emploi d'un indéfini neutre 'quelque chose' s'inscrirait dans les cas de spécification de type 'irrealis' (les non spécifiques de Haspelmath). L'interprétation FC est possible ici mais moins probable. On ne voit pas pourquoi il y aurait un sens 'ce que vous voulez' et si on avait affaire à un cas d'indifférence sur la quantification, on trouverait plutôt le choix lexical 'n'importe quoi'. Par contre, il est probable qu'il y a dans ce contexte un emploi scalaire monotone-décroissant: 'si peu que ce soit'. On a donc un choix lexical 'quoi que ce soit' qui s'inscrit dans ce cadre de la polarité faible.

$\mathrm{Si}$ on cherche à donner une règle simple de la répartition des emplois à quantification sousjacente existentielle, on dira ainsi que lorsqu'il n'y a pas scalarité, on emploie de préférence la série n'importe, alors que la série que ce soit a tendance à associer les phases de choix et d'action dans la même quantification universelle, sauf s'il y a l'interprétation à polarité associée à une orientation vers zéro de la quantification.

On trouve cependant un ensemble de données qui pourraient montrer que cette série que ce soit peut apparaître dans des contextes de quantification qui a priori ne semblent pas universels. Il s'agit toujours de construction présentant un schème syntaxique de deux prédications liées :

(41) Demandez quoi que ce soit, ça sera fait. Régalez-vous (Google)

(42) Lorsque vous demandez quoi que ce soit, veillez à utiliser des termes tels que 'Could you' et 'Please'...(Google) 
(43) Vous me demandez quoi que ce soit sur quelqu'un de l'immeuble, je sais. (Libération, 23/10/06)

(44) «Dès que l'Allemagne propose quoi que ce soit», conclut Levoir, " le quai d'Orsay déclare : «c'est un piège ! ». (R. Martin du Gard, Les Thibault, l'été 1914, 1936).

Il ne faut peut-être pas chercher à expliquer de telles constructions par la polarité négative. Ces phrases sont en réalité des conditionnelles cachées ${ }^{13}$, et le choix d'une valeur quelconque de la variable représentée par que ce soit aboutit à une conséquence qui est la seconde phrase. Il s'agit donc d'une variante dans ce type de contexte d'un emploi à quantification universelle et non existentielle (l'universalité tient à la généralisation d'un ensemble d'actions épisodiques présentées comme identiques, avec une conséquence semblable).

\section{Les emplois qualificatifs / appréciatifs}

Il s'agit d'autres constructions qui semblent assez nettement distinctes de celles rencontrées avec la série n'importe: l'emploi attributif ne fait aucun doute ici. Les exemples caractéristiques sont :

(44) Elle flirte avec n'importe qui.

(45) Tu pourrais faire attention, tu as laissé entrer n'importe qui.

(46) Nous avons bredouillé n'importe quoi et il a haussé les épaules.

(47) J'étais irrité par mon boulon et je répondis n'importe quoi.

(A. de Saint-Exupéry, Le petit prince, 1943)

(48) Vous travaillez comme une machine, et vous faites n'importe quoi.

(A. Dhôtel, Le village pathétique, 1943)

Dans le premier cas, il est théoriquement possible d'avoir une lecture en termes de quantification universelle. Cependant, le sens du prédicat et l'utilisation pragmatique plaident pour une utilisation qualificative de l'indéfini, greffée sur une quantification de type existentiel (un indéfini pluriel assez vague) plutôt qu'universel : elle flirte avec des gens qu'elle ne sélectionne pas. On peut donc poser à côté de l'interprétation à quantification une interprétation attributive du déterminant. Le second exemple s'interprète de façon identique. Dans les deux cas, on peut évidemment utiliser 'tout le monde' mais sans doute avec la même interprétation qualitative (= des gens non sélectionnés). L'utilisation de n'importe dans une phrase factive événementielle (46), (47) exclut tout autre interprétation que celle d'un indéfini singulier: quelque chose, avec la même valeur d'appréciation qualitative. En contexte habituel (phrase (48) ) le sens qualitatif est assez différent : il ne s'agit plus d'absence de sélection mais cette fois de quelque chose d'une qualité médiocre ou mauvaise.

On différenciera ces constructions de celles vues précédemment, dans lesquelles un espace de choix de type universel précède la quantification existentielle de l'action. Dans ces constructions, c'est l'inverse : la quantification soit préexiste, soit accompagne la qualification, et le déterminant semble bien être utilisé de façon attributive pour qualifier cette quantification existentielle sous-jacente non précisée, avec des interprétations qui vont de l'absence de sélection à la mauvaise qualité.

\section{Bilan}

\footnotetext{
${ }^{13}$ Les conditions sont assez proches de l'emploi concessif basique de cette série, qui correspond aux mêmes contraintes sémantiques : quoi que ce soit qu'on me demande, je sais répondre.
} 
Les séries FC ne sont pas directement aptes à signifier la quantité d'individus qui ont une fonction d'argument pour un prédicat quelconque. Leur sens propre, qui bloque les opérations de spécification, devrait aboutir à signifier la quantification universelle, sens qu'on retrouve effectivement, soit dans les énoncés génériques, soit dans certains énoncés événementiels. Pourtant, on les emploie dans quantité de contextes dans lesquels la quantification n'est pas universelle. Pour décrire ces emplois, on a parlé de quantification dissociée : l'indéfini de libre choix signale le plus souvent la possibilité illimitée du choix qui précède l'action, et la quantification de l'action est régie par la pragmatique de la communication et les présupposés attachés l'emploi de tel ou tel prédicat. Dans les phrases dénotant un succession d'actions identiques, ou dans les énumérations, les indéfinis de libre choix peuvent signifier une forme de généralisation à l'infini, une somme d'actions dans lesquelles un protagoniste quelconque dont l'identité importe peu est impliqué, si bien qu'on peut le présenter comme interchangeable : le n'importe qu- de fin de séquence n'est pas très loin du etc... des fins d'énumérations. Avec la négation, le libre choix est naturellement lié à l'un des aspects essentiels de la sémantique de ce contexte, la quantification universelle associée à un prédicat négatif pour signifier la quantité nulle. Dans les contextes à polarité, notamment en dehors des phrases négatives, il arrive souvent que l'indéfini de libre choix soit associé à des présupposés négatifs sur l'existence de son référent, même lorsque celle-ci est avérée dans le contexte. Le phénomène de dissociation est alors entre l'univers des présupposés et celui qui envisage le phénomène comme réel. Assez différent est l'emploi qu'on a nommé "qualificatif» des indéfinis de libre choix : il se présente cette fois comme une appréciation a posteriori d'un terme à valeur existentielle, le plus souvent spécifié, mais jugé comme peu pertinent, ou déprécié d'une façon ou d'une autre.

Comme on l'a vu sur de multiples exemples, les séries de libre choix ne sont pas interchangeables dans ces emplois : il est assez difficile de dissocier choix et quantification réelle avec que ce soit, sauf dans les contextes à polarité. En revanche, aussi bien dans les emplois où la phase du choix précède celle de l'action que dans ceux où l'indéfini qualifie a posteriori, la série n'importe permet assez largement cette dissociation sur de nombreux contextes.

\section{Références}

CARLSON G. (1981), "Distribution of Free-Choice Any" in Papers from the Seventeenth Regional Meeting of the Chicago Linguistics Society, Chicago : CLS , 17, University of Chicago, p. 8-23.

DAYAL V. (2004), "The Universal Force of Free-Choice Any, "Linguistic Variation Yearbook 4, 5-40.

FAUCONNIER G. (1975), "Pragmatic Scales and Logical Structures”, Linguistic Inquiry, 6, 353-375.

Haspelmath M. (1997), Indefinite Pronouns, Oxford : Oxford University Press.

HoRN L. R. (2000), "Pick a Theory (Not Just Any Theory)", in Negation and Polarity, L.R. Horn \& Y. Kato (eds), Oxford : Oxford U.P., p. 147-192.

JAYEZ J. \& L. TOVENA (2005), "Free Choiceness and Non-Individuation", Linguistics and Philosophy, 28, p. 171.

KADMON N. \& F. LANDMAN (1993), "Any", Linguistics and Philosophy, 16, p. 353-422.

LADUSAW W.A. (1980), Polarity Sensitivity as Inherent Scope Relations, New York : Garland.

MARTIN R. (1992), Pour une logique du sens, Paris : PUF.

MUlLER C. (2006), "Polarité négative et free choice dans les indéfinis de type que ce soit et n'importe », Langages, 162 , p. 7-31.

MULLER C. (2007), «Les indéfinis free choice confrontés aux explications scalaires », Travaux de linguistique, 54, p. 83-96

MULLER C. (2008), «Valeurs communes et valeurs particulières des formes Qu- en français », Langue Française, 158, 13-28.

MULLER C. (2009), «Une cartographie des indéfinis free choice du français », Syntaxe et Sémantique, 10, p. 6578.

PAILlARD D. (1997), « N'importe qui, n'importe quoi, n'importe quel N », langue Française, 116, p. 100-114. VENDLER Z. (1967), Linguistics in Philosophy, Ithaca, NY : Cornell University Press. 
Vlachou E. (2007), Free Choice in and out of Context: Semantics and Distribution of French, Greek and English Free Choice Items, Utrecht : LOT. 